\title{
Mass transfer with reaction kinetics of the biocatalytic membrane reactor using a fouled covalently immobilised enzyme layer ( $\alpha$-CGTase-CNF layer)
}

\begin{abstract}
A dynamic mathematical model was used in this study to describe the mass transfer and reaction kinetics of a fouled $\alpha-$ CGTase-CNF layer in a biocatalytic membrane reactor (BMR) system. BMR performance was evaluated based on the effect of substrate concentration and pneumatic gauge pressure on the permeate flux, total permeate volume, starch conversion, and $\alpha$-CD production. A model is introduced based on the layer of mass limit as the resistance blocking mechanism with reaction kinetics on the fouled $\alpha$-CGTase-CNF layer. Important unknown parameters of the constants describing the layer resistance, KML0 and the kinetic constant of Michaelis-Menten, kcat, for mass transfer and its reaction kinetics, were successfully estimated at $3.9 \times 107 \mathrm{~min} / \mathrm{mL}$ and $216.18 \mathrm{mg}$-starch/cm3 $3 \cdot \mathrm{U} \cdot \mathrm{min}$, respectively, using the proposed models. The study found that the kcat value corresponding to the maximum efficiency of the enzyme on starch cyclisation was a critical parameter in the prediction of the BMR dynamic profiles. Thus, the proposed model in this study can be used for further development of the BMR system, with excellent hydrodynamic and reaction performance.
\end{abstract}

Keyword: Microalgal diatom; Metabolic profiles; Algalomics; Chemical markers; UHPLCMS 